\title{
Clinical, radiological and polysomnographic evaluation of sleep disordered breathing in children
}

\author{
Shikha Bhool, J. C. Suri, M. K. Sen, V. P. Venkatachalam \\ Department of Pulmonary, Critical Care \& Sleep Medicine and ENT, Vardhman Mahavir Medical College \& \\ Safdarjang Hospital, New Delhi
}

Indian J Sleep Med 2008; 3.3, 85-92

\begin{abstract}
Introduction: There is very little data of Indian children with sleep disordered breathing (SDB), regarding the common clinical presentation, etiological factors, radiological and polysomnographic features. We therefore carried out a study to evaluate these factors in Indian children presenting to a tertiary care hospital.

Material And Method: Forty consecutive children were referred from the ENT outpatient for overnight polysomnography because of symptoms suggestive of sleep disordered breathing (SDB). A complete general, physical and systemic examination, including height, weight, BMI, was conducted. ENT examination was performed to include craniofacial characteristics such as mid face hypoplasia, micrognathia and occlusal relationships. Radiological examination of the airway was performed. Digital skiagram of soft tissue neck in lateral view was obtained and A-N ratio (adenoid:nasopharynx ratio), H-MP (H-MP = distance from mandible to hyoid) and PAS (PAS = Posterior airway space) were assessed.

Results: The age of the children varied from 1 to 15 years. A male preponderance was noticed (72.5\%). Maximum number of the children was underweight (82.5\%). All the children complained of snoring and mouth breathing. The other symptoms noted were disturbed sleep (65\%); frequent cold and difficult swallowing (62.5\%). Poor school performance was seen in $30 \%$; Enuresis in $22.5 \%$ and aggressive behaviour in $7.5 \%$. Analysis of the behavioural symptoms showed that $30 \%$ of the children had poor school performance, $22.5 \%$ had bedwetting, $7.5 \%$ had aggressive behaviour, $2.5 \%$ each had sleep terror and sleep walking respectively. The upper airway examination (clinical and endoscopic) showed that adenotonsillar enlargement was the most common cause of upper airway obstruction among these children. The other findings were adenoid enlargement $(7.5 \%)$, tonsil enlargement $(22.5 \%)$, deviated nasal septum $(2.5 \%)$, deviated nasal septum with adenoid $(2.5 \%)$, polyp $(7.5 \%)$ and rhinitis $(2.5 \%)$. Cephalometric analysis showed that The S-PAS value in controls was $15-20 \mathrm{~mm}$ whereas that of the cases was only $2-5 \mathrm{~mm}$. The distance from mandible to hyoid (H-MP) value was also found to be increased, thus indicating a low position of the mandible in $67.5 \%$ of cases. $\mathrm{A}: \mathrm{N}$ ratio ( $\mathrm{A} \mathrm{N}$ ratio=adenoid:nasopharynx) was abnormal in $65 \%$ of the children. Polysomnography showed that $60 \%$ children had severe SDB (RDI>10 per hour) and $30 \%$ of them had moderately severe SDB (RDI 5-10/hr). About $50 \%$ children showed significant oxygen desaturation.

Conclusions: In conclusion it can be said that every child who snores is a suspected case of SDB. So there should be proper evaluation and management at the earliest to prevent potentially serious complications.
\end{abstract}

Keywords: SDB, children, PSG, India

Address for correspondence:

Dr. J. C. Suri

Senior Chest Physician \& Head

Department of Pulmonary, Critical Care \& Sleep

Medicine, Safdariang Hospital, New Delhi

E-mail: jcsuri@rediffmail.com 


\section{Introduction}

S leep-disordered breathing is a commonly encountered health problem and is usually overlooked. It encompasses a continuum of upper airway obstructing ranging from primary snoring to more serous obstructive sleep apnea syndrome. The prevalence of primary habitual snoring among all children is between $8 \%$ to $12 \%$ and that of OSA between $1 \%$ and $4 \%$. (1). In a recently published study by the author, the prevalence of snoring and SDB in North Indian school children was found to be $11.6 \%$ and $6.1 \%$ respectively (2). SDB is known to be associated with significant morbidity in the form of impairment of physical growth, developmental delay, behavioral disturbances and poor school performance. (3),(4),(5) The most common causes of OSAS in children are related to upper airway obstruction due to adeno-tonsillar hypertrophy and other cranio-facial abnormalities. However, with the increasing prevalence of obesity in children, the latter is also becoming an important causative factor for OSA in this population. There is very little data of Indian children regarding the common clinical presentation, etiological factors and polysomnographic features. We therefore carried out a study to evaluate these factors in Indian children presenting to a tertiary care hospital.

\section{Material and Method}

Forty consecutive children were referred from the ENT outpatient department to the Department of Pulmonary, Critical Care \& Sleep Medicine for overnight polysomnography because of symptoms suggestive of sleep disordered breathing (SDB). These symptoms included snoring, mouth breathing, recurrent upper respiratory infections, nocturnal choking, witnessed apneas, enuresis, frequent awakenings or arousals. These children were then asked to fill-up a questionnaire which included common questions related to SDB, upper airway obstruction (tonsils, adenoids), sleep habits, and parentally observed abnormalities of behaviour, learning and development. Following a detailed analysis of symptoms, meticulous clinical examination was carried out. A complete general, physical and systemic examination, including height, weight, BMI, was conducted. ENT examination was performed to include craniofacial characteristics such as mid face hypoplasia, micrognathia and occlusal relationships. Septal deviation, choanal atresia, nasal polyps and turbinate hypertrophy were also looked for. Oral cavity was examined for the shape and height of the palate, crowding of teeth, size of tongue, tonsils and uvula. Tonsillar size was graded as grade I (tonsils in tonsillar pillar) grade II, (tonsils protruding from tonsillar pillar), grade III (tonsils reaching midpoint between the anterior-pillar \& uvula) and grade IV (tonsils reaching the uvula). Mallampati scoring was performed as follows: Grade I (Palatal arches $\&$ uvula are fully visible), Grade II (Palatal arches are partly visible \& uvula is hidden by tongue) and Grade III (Only the soft palate could be visualized). ${ }^{(17)}$ Radiological examination of the airway was performed. Digital skiagram of soft tissue neck in lateral view was obtained and A-N ratio (adenoid:nasopharynx ratio), H-MP (H-MP = distance from mandible to hyoid) and PAS (PAS= Posterior airway space) were assessed. Since there was insufficient data about the normal cephalometric values in Indian children in the literature, five normal children (with no SDB) were randomly selected from each of the three age groups were taken as controls to obtain the normal values. These cephalometric parameters provide an assessment of the dimensions of the upper airway.

SDB was confirmed in these children by polysomnography. Whole night polysomnography was performed (Alice 5;Respironics; Murrysville, PA) for 6 to 7 hours by a trained technologist. The following parameters were measured: 2 channels each for electroencephalography (EEG); electro-oculography (EOG); electro-myography (EMG) with submental electrodes; electro-cardiography (ECG); airflow recording through nose and mouth by a thermistor and nasal pressure cannula; thoracic and abdominal efforts by plethysmography; oxygen saturation through pulse oximetery; and tracheal sound recording by using a microphone attached to the neck.

The polysomnography criteria used were as follows. Obstructive apnea was defined as cessation of airflow at the nose and mouth, as measured by thermistor/ nasal cannula while the respiratory effort continues for at least two breaths. Hypopnea was defined as more that or equal to $50 \%$ decrease in the amplitude of the nasal/oral airflow signal accompanied by hypoxemia or arousal. Clinically significant hypoxemia was diagnosed by the presence of any event associated with oxyhemoglobin desaturation greater than $4 \%$ from the baseline $\mathrm{SpO}_{2}$ or any $\mathrm{SpO}_{2}$ value less that or equal to $92 \%$. Snoring children with an apnea-hypopnea index (AHI) of less than or equal to one per hour of total sleep time (TST) with no clinically significant hypoxemia, hypercapnia or 
excessive daytime sleepiness were considered to have primary snoring. ${ }^{(16)}$ Respiratory effort related arousals (RERA) was diagnosed when a series of breaths lasting for at least 10 seconds showing flattening or airflow limitation on nasal pressure waveform were followed by an arousal from sleep. SDB was diagnosed when a patient with symptoms suggesting SDB had an RDI (apneas + hypopneas + RERAs) was $>1$ per hour.

\section{Statistical Methods}

All the data analysis was performed using statistical software SPSS 11.0. Chi-square test and t-test were used to determine significance of correlation and testing the differences in the prevalences by characteristics

\section{Results}

The age of the children varied from 1 to 15 years. The majority of children presenting to the OPD were between 6 to 10 years (mean age 8 years). (Table-1)

Table 1: Age distribution of patients

\begin{tabular}{|l|l|c|}
\hline Age (years) & No. of children $(\mathbf{n}=\mathbf{4 0})$ & Percentage (\%) \\
\hline $1-5$ & 10 & 25 \\
\hline $6-10$ & 20 & 50 \\
\hline $11-15$ & 10 & 25 \\
\hline
\end{tabular}

A male preponderance was noticed $(72.5 \%)$. The morphometric distribution showed that maximum number of the children was underweight $(82.5 \%)$. The others were normal, healthy children. None were found to be overweight or obese. (Table-2)

Table 2: Body mass index (BMI) of the patients

\begin{tabular}{|c|c|c|}
\hline BMI & No. of children $(\mathbf{n}=40)$ & Percentage \\
\hline$<18.5$ & 33 & 82.5 \\
\hline $18.5-24.9$ & 7 & 17.5 \\
\hline
\end{tabular}

All the children complained of snoring and mouth breathing. (Table3). The other symptoms noted were disturbed sleep (65\%); frequent cold and difficult swallowing $(62.5 \%)$. Poor school performance was seen in $30 \%$; Enuresis in $22.5 \%$ and aggressive behaviour in $7.5 \%$.

Analysis of the behavioural symptoms showed that $30 \%$ of the children had poor school performance, $22.5 \%$ had bedwetting, $7.5 \%$ had aggressive behaviour, $2.5 \%$ each
Table 3: Symptoms of the patients

\begin{tabular}{|l|l|l|}
\hline Symptoms & $\begin{array}{l}\text { No. of children } \\
(\mathbf{n}=40)\end{array}$ & percentage \\
\hline Snoring & 40 & 100 \\
\hline Mouth breathing & 40 & 100 \\
\hline Frequent cold & 25 & 62.5 \\
\hline Enuresis & 9 & 22.5 \\
\hline Disturbed sleep & 26 & 65 \\
\hline Difficulty in swallowing & 25 & 62.5 \\
\hline
\end{tabular}

had sleep terror and sleep walking respectively (Table 4)

Table 4: Behavioral symptoms $(n=40)$ in the patients

\begin{tabular}{|l|c|c|}
\hline Symptoms & Results & Result (\%) \\
\hline $\begin{array}{l}\text { Poor School } \\
\text { Performance }\end{array}$ & 12 & $30 \%$ \\
\hline Aggressive Behavior & 3 & $7.50 \%$ \\
\hline Sleep terrors & 1 & $2.50 \%$ \\
\hline Sleep walking & 1 & $2.50 \%$ \\
\hline
\end{tabular}

The upper airway examination (clinical and endoscopic) showed that adenotonsillar enlargement was the most common cause of upper airway obstruction among these children. (Table 5). The other findings were adenoid enlargement $(7.5 \%)$, tonsil enlargement $(22.5 \%)$, deviated nasal septum $(2.5 \%)$, deviated nasal septum with adenoid (2.5\%), polyp (7.5\%) and rhinitis $(2.5 \%)$

Table 5: Upper airway examination findings

\begin{tabular}{|l|l|l|}
\hline Observation & $\begin{array}{l}\text { No. of children } \\
(\mathbf{n}=40)\end{array}$ & Percentage \\
\hline $\begin{array}{l}\text { Adeno-tonsillar } \\
\text { enlargement }\end{array}$ & 22 & 55 \\
\hline Adenoid enlargement & 3 & 7.5 \\
\hline Tonsil enlargement & 9 & 22.5 \\
\hline Deviated nasal septum & 1 & 2.5 \\
\hline $\begin{array}{l}\text { Deviated nasal septum } \\
\text { with adenoid }\end{array}$ & 1 & 2.5 \\
\hline Polyp & 3 & 7.5 \\
\hline Rhinitis & 1 & 2.5 \\
\hline
\end{tabular}

Among these children, three had cranio-facial anomaly with adenoid enlargement as well like Treacher-

Indian Journal of Sleep Medicine (IJSM), Vol. 3, No. 3, 2008 
Collin syndrome, retrognathia and prognathia.

Clinical examination of the oral cavity showed that $75 \%$ of the children had high arched palate: $65 \%$ had crowding of teeth and $7.5 \%$ had adenoid facies (Table 6). Analysis of Mallampati grading showed that 16 patients had Mallampati grade 3 and 14 and 10 patients had grade II and grade I respectively (Table 7)

Table 6: Craniofacial Anomalies $(\mathrm{n}=40)$

\begin{tabular}{|l|l|l|}
\hline Symptoms & Results & Result (\%) \\
\hline High Arched Palate & 30 & $75 \%$ \\
\hline Crowded teeth & 26 & $65 \%$ \\
\hline Retrognathia & 1 & $2.50 \%$ \\
\hline Prognathia & 1 & $2.50 \%$ \\
\hline Long Uvula & 1 & $2.50 \%$ \\
\hline Large bulky tongue & 1 & $2.50 \%$ \\
\hline Adenoid facies & 3 & $7.50 \%$ \\
\hline
\end{tabular}

Table 7: Mallampati Grade

\begin{tabular}{|l|l|}
\hline Grade I & 10 \\
\hline Grade II & 14 \\
\hline Grade III & 16 \\
\hline Grade IV & None \\
\hline
\end{tabular}

\section{Cephalometric data}

The average width of the pharyngeal airway at the most postero-superior point on the soft palate (S-PAS) value of control was compared with that of the cases. The $S$ PAS value in controls was $15-20 \mathrm{~mm}$ whereas that of the cases was only $2-5 \mathrm{~mm}$. The distance from mandible to hyoid (H-MP) value was also found to be increased, thus indicating a low position of the mandible in $67.5 \%$ of cases. (Table 8).

Table 8: Cephalometric Analysis $(n=40)$

\begin{tabular}{|l|c|c|}
\hline H-MP distance $(\mathrm{mm})$ & No. of Children & Percent \\
\hline Normal ( $14.8-15.9 \mathrm{~mm})$ & 13 & 32.5 \\
\hline Increased (Low positioned hyoid) & 27 & 67.5 \\
\hline
\end{tabular}

$\mathrm{H}-\mathrm{MP}=$ distance from mandible to hyoid

$\mathrm{S}-\mathrm{PAS}=$ Posterior airway space

Radiological analysis also showed that A: $\mathrm{N}$ ratio (A $\mathrm{N}$ ratio=adenoid:nasopharynx) was abnormal in $65 \%$ of the children. The distribution of severity of adenoid enlargement is is depicted in Table 9.

Polysomnography showed that $60 \%$ children had
Table 9: Radiological Analysis A : $\mathrm{N}$ ratio

\begin{tabular}{|l|c|c|}
\hline $\mathrm{A}: \mathrm{N}$ & No. of Children & Percent \\
\hline $0.5 \pm 0.1$ (Normal & 14 & 35 \\
\hline $0.6 \pm 0.1$ (Mild) & 13 & 32.5 \\
\hline $0.7 \pm 0.1$ (Moderate) & 9 & 22.5 \\
\hline 0.8 (usually enlarge) & 4 & 10 \\
\hline
\end{tabular}

severe SDB (RDI>10 per hour) and $30 \%$ of them had moderately severe SDB (RDI 5-10/hr). About 50\% children showed significant oxygen desaturation. (Table 10)

Table 10: Severity of SDB on polysomnography

\begin{tabular}{|c|c|c|c|}
\hline RDI & \multicolumn{2}{|c|}{ No. of Children } & Percent \\
\hline $1-4($ mild $)$ & \multicolumn{2}{|l|}{4} & 10.0 \\
\hline 5-10 (moderate) & \multicolumn{2}{|c|}{12} & 30.0 \\
\hline$>10$ (severe $)$ & \multicolumn{2}{|c|}{24} & 60.0 \\
\hline \multicolumn{2}{|l|}{$\mathrm{S}_{\mathrm{a}} \mathrm{O}_{2}$} & No. of Children & Percent \\
\hline \multicolumn{2}{|l|}{$>92 \%$ Normal } & 20 & 50 \\
\hline \multicolumn{2}{|c|}{$<92 \%$ Hypoventilation } & 20 & 50 \\
\hline
\end{tabular}

Patients with predominantly pharyngeal obstruction (adeno tonsillar or tonsillor hyper trophy) had more severe SDB than those with nasal obstruction (DNS, antro-choanal polyp) (Table 11).

Table 11: Severity of SDB in relation to clinical / endoscopy findings

\begin{tabular}{|l|c|c|c|}
\hline Clinical & No. of Children & Avg. RDI & Agv. $\mathrm{S}_{\mathrm{a}} \mathrm{O}_{2}$ \\
\hline Adenotonsillar hypertrophy & 20 & 25 & 87 \\
\hline Adenoid & 3 & 19.8 & 93.6 \\
\hline Tonsil & 9 & 18.3 & 90.5 \\
\hline DNS & 4 & 3 & 98 \\
\hline DNS with Adenoid & 1 & 45 & 90 \\
\hline Polyp (antro-choanal) & 3 & 27 & 93.3 \\
\hline
\end{tabular}

\section{Discussion}

This study was conducted primarily to determine the clinical, etiological and morphological profile of upper airway obstruction in children with sleep disordered breathing who were less than 15 years of age. Fifty percent 
of children in this study were between 6 and 10 years. It may be ascribed to the occurrence of the peak incidence of adeno-tonsillar hypertrophy in this age group.(7) Twenty five percent of the children were between 1 and 5 years of age. This has important long term implications on the physical and mental growth of the child as about $60 \%$ of the adult face is already built up by the age of four years and the adeno-tonsillar enlargement in this age may significantly interfere with this growth. According to most epidemiological studies gender appears to play no significant role in pediatric SDB. $(27,28)$ Similar results were found in the epidemiological study of sleep disorders in Indian school going children that was conducted by one of the authors.(2) In this study we found a significant male preponderance of patients (males $=72.5 \%$ ) which was also in another study (8). The gender bias of SDB seen among children seeking medical attention may be ascribed to the common attitude of lack of attention to the girl child that is seen in the Indian scenario.

Obesity is an important risk factor for SDB in adults, but has not found to be an important factor in pediatric sleep apnea. $(29,30)$ However with the increasing prevalence of obesity in childhood, it may be important in some cases of OSA in children. A recent review has proposed that two types of OSA disease in children may exist. The first category (type-I) is associated with marked lymphadenoid hypertrophy in the absence of obesity. The other category (type II) is primarily associated with obesity in the presence of only mild lymphadenoid hyperplasia (25). Failure to thrive and impaired growth are frequently seen in children with SDB which significantly improves after adenotonsillectomy. $(31,32)$ The most common cause of failure to thrive is believed to be an increase in work of breathing resulting into a rise in baseline caloric expenditure.(13) Somatic growth can also be impaired due to decreased appetite, dysphagia due to tonsillar enlargement, decreased levels of insulin growth factor1 , insulin growth factor binding protein and possibly growth hormone $(19,20)$. Most of the children in this study were under weight $(82.5 \%)$ indicating failure to thrive. Obesity /overweight were non-contributory to the occurrence of SDB in the study population. It may also be mentioned that the absence of obese children in this study may also be attributed to the fact that they were derived from the OPD of a public sector Government hospital which caters usually to patients from lower socio-economic strata. A larger study, involving multiple centers covering all socio-economic strata, may be more helpful in clarifying the contribution of obesity in the causation of SDB in Indian children.

Snoring is the most frequent presenting feature in children with OSA. In a study of children presenting to a sleep clinic in Hong Kong, snoring was found to be the single most significant risk factor.(21) Mouth breathing has been found to have a sensitivity of 29 to $78 \%$ and a specificity of $27-56 \%$ in the diagnosis of OSA in various studies. $(29,33,34,35)$ In our study both snoring and mouth breathing were universally seen in all the patients ( $100 \%$ sensitivity), thus indicating that these are very important high risk factors of SDB. Nocturnal enuresis has been found to be more prevalent in OSA patients $(47 \%)$ as compared to non OSA children (17\%).(36) Another study found that nocturnal enuresis was significantly high (51.6\%) in OSA children compared to only $15.8 \%$ in primary snorers.(26) Enuresis had the highest predictive accuracy in a study of 82 children with OSA.(34) Enuresis was observed in $22.5 \%$ of the children in the present study as against $7.7 \%$ in the general population of children in the community based survey.(2) In children with OSA, behavioral and cognitive problems are very common.(37) In the present study about one third of the patients had poor school performance and $7.5 \%$ had aggressive behavior. Behavioral and cognitive problems along with poor school performance have also been frequently seen in children with OSA in several other studies. $(22,23,24,37)$ They were found to improve after treatment of OSA.(38) Poor school performance could result from sleep fragmentation caused by intermittent hypoxia accompanied with frequent arousals from sleep.(39) The presence of all the above mentioned clinical features in children, thus, should raise a strong suspicion of SDB in children of this age group.

The majority of children (85\%) in this study had upper airway obstruction due to enlargement of adenoid and tonsils. The remaining 15\% had other causes such as deviated nasal septum (DNS), nasal polyps and rhinitis.

High-arched palate (75\%), crowding of teeth (65\%) and narrow angulation of maxilla were observed in our patients.(Table 5-7)Another recent study has also observed that compared to non-obstructed controls, the OSAS children exhibited a narrow dental arch, high frequency of lateral crossbite, and a reduced overbite. These patients also demonstrated a large lower anterior 
face height, a posteriorly inclined mandible and facial axis, and retroclined incisors. ${ }^{(14)}$ These cranio-facial features are highly suggestive of anatomically small pharynx and oral cavity in majority of the children. Thus there was an evidence of both intra-luminal obstructive lesions (adeno-tonsillar hypertrophy, or DNS, or polyps, or rhinitis) as well as an anatomically small pharynx in most of the children in the study population. This was further substantiated in our patients by the cephalometric findings of decreased S-PAS value and increased H-MP values as compared to the controls. Similar cephalometric findings have also been reported in other studies.(9-12) The simultaneous presence of adeno-tonsillar enlargement and the anatomically small pharyngeal air space may be suggestive of an inter-relationship between them. It has been suggested that the presence of nasal obstruction early in life can result into developmental abnormalities of cranio-facial structures. In an experiment performed in newborn monkeys, Harvold et al demonstrated that a gradual occlusion of the nasal airway led to morphological changes in the naso-maxillary complex, mandible and the pharyngeal air space. These included narrowing of the dental arches, decrease in maxillary arch length, an anterior crossbite, maxillary overjet and an increase in anterior face height. Such changes are associated with SDB and may be induced by altered function of the muscles such as genioglossus and loss of contribution of the tongue in the development of maxillary arch as a result of mouth breathing. (40) Thus the adeno-tonsillar enlargement in most of our patients early in life may have resulted into various cranio-facial abnormalities (high-arched palate, overcrowding of teeth, maxillary overjet) leading to anatomically small oral and pharyngeal air space. An early removal of hypertrophied adenoids and tonsils may prevent the development of such abnormal changes.

Overnight polysomnography (PSG) is the gold standard test for the diagnosis of childhood OSA. However there are difficulties in performing and interpreting PSG in children. The parameters originally used to evaluate pediatric PSG were based on adult values of OSA. Because of children's low lung reservoir and a higher baseline respiratory rate, clinically relevant apneas may be much shorter in duration (i.e 3 to 4 seconds). Two recent studies have suggested that $\mathrm{AHI}>1$ and $\mathrm{SpO} 2$ $<92 \%$ should be considered as abnormal in children $(16,41)$. The use of apnea - hypopnea index
(AHI) for the diagnosis of OSA in children may be inappropriate as many children demonstrate episodes of prolonged hypoventilation with few discrete apneas and hpopneas. $(42,43,44)$ In a study involving 213 children, 2 to 7 years of age with PSG evidence of SDB, only $24 \%$ had OSA based on AHI frequency at least 15 event per hour.(44) Many children show repeated arousals from the sleep (RERA) due to increase in the breathing effort without any evidence of frank apnea or hypopnea on PSG. (42) The increased effort can be demonstrated on a PSG as flow limitation on nasal pressure recording or by esophageal pressure monitoring. Our study showed that all the children who were clinically suspected to have OSA had significant SDB on PSG. About $50 \%$ of the children had significant oxygen desaturation $(\mathrm{SpO} 2<92 \%)$. This indicates that selection criteria (that were used based on symptoms, clinical findings, cephalometric and endoscopic findings) were highly predictive of significant SDB as confirmed later by polysomnography. It was also seen that those patients who had obstruction at the level of pharynx (adenotonsillar/ tonsillar hypertrophy) had more severe SDB than those who had obstruction at the nasal level (antrochoanal polyps, deviated nasal septum) (Table 11).

On evaluation of the polysomnographic data it was found that frank apneas were seen in only one third of the patients and they were of short duration and the majority of the children in this study either showed hypopneas or demonstrated periods of flow limitation followed by EEG arousals (RERA). This highlights the importance of the use of either oesophageal or nasal pressure monitoring in the detection of these events which can be otherwise overlooked or missed with the standard airflow monitoring by nasal thermisters.

Also, in the overall evaluation of SDB, the various outcome measures like poor school performance, aggressive behaviour, and somatic growth retardation should be taken into consideration while making decisions about the treatment of these children.

In conclusion it can be said that every child who snores is a suspected case of SDB. Combining clinical and radiological findings have been found to be helpful in screening children with clinically significant OSA who need earlier investigation and intervention. After the diagnosis is confirmed with PSG these children should undergo adeno-tonsillectomy and other procedures at the earliest to prevent potentially serious complications. 


\section{References}

1. Ali NJ, Pitson DJ, Stradling JR. Snoring, sleep disturbance and behaviour in 4-5 year olds. Arch Dis Child 1993; 68: 360-366.

2. Suri JC, Sen M.K. Epidemiology of sleep disorders in schoolchildren of Delhi: a questionnaire-based study. Indian J sleep Med 2008;3(2). (in Press)

3. Schechter MS. Section on pediatric pulmonology, subcommittee on obstructive sleep apnea syndrome. Technical report : diagnosis and management of childhood obstructive sleep apnea syndrome. Pediatrics 2002; 109 : e69.

4. Owen GO, Canter RJ, Robinson A. Snoring, apnea and ENT symptoms in the paediatric community. Clin otolaryngol 1996; $21: 130-4$.

5. Katz ES, Marcus CL. Diagnosis of obstructive sleep apnea syndrome in infants \& children. In : Sheldon SH, Ferber $\mathrm{R}_{\text {, }}$ Kryger MH, editors. Principles \& practice of Pediatric sleep medicine. USA. Elseirer Saunders; 206 197-210.

6. Section on Pediatric Pulmonology, subcommittee on OSAS American academy of pediatrics Clinical practice guidelines: diagnosis and management of childhood OSAS, Pediatrics 109; 2002, 704-712

7. Sher AE : OSAS : a complex disorder of the upper airway. Otolaryngol Clin North Am 23 : 593-608, 1990.

8. Chaudhary BA, Speir WA. Sleep apnea syndrome. South Med J 1982;75:39-45.

9. Acebo $\mathbf{C}$, Millman RP, Rosenberg $\mathrm{C}$ et al. Sleep, breathing and cephalometrics in older children \& young adults. Part 1 Normotensive values. Chest 1996; 109: 664-672.

10. Shintani T, Asakura K, Katawra A. OSA in Children. Advance Otorhinolaryngol (1992), 47; 267-270.

11. Miles PG, Uig PS, Weijant RJ et al. Cranio facial structure and OSAS : a qualitative analysis of the literature. Am J Orthod Dentofacial Orthop 1996; 109: 163-172.

12. Pracharktam $M$, Hans $M G$, Stohl $K P$ et al. Upright \& supine cephalometric evaluation of OSAS \& snoring subjects. Angle Orthod 1994; 64 : 63-74.

13. Chan J, Edman JC, Kolsai PJ : OSA in children. Am Fam Physician 2004; 69 : 1147-1154.

14. Wijk L. Z. A longitudinal study of dento-facial morphology in young children treated for obstructive sleep apnea syndrome. Karolinska Institutet, Stockholm 2008:1-6.

15. Wagner $\mathbf{M H}$, Torrez DM.Interpretation of the polysomnogram in children. Otolaryngol Clin N Am 2007; 40:745-759.

16. Uliel S, Tauman R, Green Field $M$ et al Normal polysomnographic respiratory values in children and adolescents. Chest 2004;125:872-878

17. Mallampati SR, Gatt SP, Gugino LD, Desai SR, Waraksa B, Freiberger $D$, et al. A clinical sign to predict difficult tracheal intubation : a prospective study Can Anaesth. Soc J 1985; 32 : 429-434.

18. Arens R, Marcus CL. Pathophysiology of upper airway obstruction: a developmental perspective. Sleep 2004; 27 : 997-1019.

19. Bar A, Tarasiuk A, Seger $Y$ et al. The effect of adenotonsillectomy in serum insulin - like growth factor I and growth in children with OSAS. J Pediatr 1999; 135: 76-80.

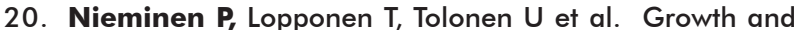
biochemical markers of growth in children with snoring and OSA. Pediatrics 2002;109:e55.

21. Chau KU, Ng DKK, Kwak CKL, Chow PY, Ho JCS. Clinical risk factors for obstructive sleep apnea in children. Singapore Med J 2003;44:570-573.

22. Chervin RD, ARchibold KH. Hyperactivity and polysomnographic findings in children evaluated for sleepdisordered breathing. Sleep $2001 ; 24: 313-320$.

23. O'Brien LM, Mervis CB, Holbrook CR et al. Neurobehavioural implications of habitual snoring in children. Pediatrics 2004;114:44-49.

24. O'Brien LM, Gozal D. Sleep in children with attention deficit/hyperactivity disorder. Minerva Pediatr 2004; 56: 585-601

25. Dayyat E, Kheirandish-Gozal L. Childhood obstructive sleep apnea: one or two distinct disease entities? Sleep Med Clin 2007; 2: 433-444.

26. Zhifei $\mathbf{X}$, Cheuk DKL, Lee SL. Clinical evaluation in predicting childhood obstructive sleep apnea. Chest 2006;130:6: 1765-1771.

27. Teculescu DB, Caillier I, Perrin $\mathrm{P}$, et al: Snoring in French preschool children. Pediatr Pulmonol 1992;13:239-244.

28. Hulcrantz E, Lofstarnd TB, Ahlquest RJ: The epidemiology of sleep related breathing disorders in children. Int $J$ Pediatr Otorhinolaryngol 1995;6(suppl):S63-S66.

29. Carroll JL, McColley SA, Marcus CL, et al. Inability of clinical history to distinguish primary snoring from obstructive sleep apnea syndrome in children. Chest 1995; 108: 610-618.

30. Leach J, Olson J, Hermann J, et al. Polysomnographic and clinical findings in children with obstructive sleep apnea. Arch Otolaryngol Head Neck Surg 1992;118:741-744

31. Lind MG, Lundell BP, Tonsillar hyperplasia in children. A cause of obstructive sleep apneas, $\mathrm{CO} 2$ retention, and retarded growth. Arch Otolaryngol 1982;108:650-4.

32. Freezer NJ, Bucens IK, Robertson CF. Obstructive sleep apnoea presenting as failure to thrive in infancy. J Paediatr Child Health 1995;31:172-5

33. Nieminen $\mathbf{P}$, Tolonen $U$, Lopponen $H$. Snoring and obstructive sleep apnea in children: a 6-month follow-up study. Arch Otolaryngol Head Neck Surg 2000;126:481486

34. Wang RC, Elkins TP, Keech D, et al. Accuracy of clinical evaluation in pediatric obstructive sleep apnea. Otolaryngl Head Neck Surg 1998;118:741-744

35. Preutthipan A, Chantorojanasiri T, Suwanjutha $S$, et al. Can parents predict the severity of childhood obstructive sleep apnoea? Acta Paediatr 2000;89:708-712.

Indian Journal of Sleep Medicine (IJSM), Vol. 3, No. 3, 2008 
36. Brooks LJ, Topol HI. Enuresis in children with sleep apnea. $J$ pediatr 2003;142:515-518.

37. Blunden S, Lushington K, kennedy D, Martin J, Dawson D. Behavior and neurocongnitive performance in children aged 5-10 years who snore compared to controls. J Clin Exp Neuropsychol 2000;22:554-68.

38. Gozal D, Pope DW Jr. Snoring during early childhood and academic performance at ages thirteen to fourteen years. Pediatrics $2001 ; 107: 1394-9$.

39. Owens J, Opipari L, Nobile C, Spirito A. Sleep and daytime behavior in children with obstructive sleep apnea and behavioral sleep disorders. Pediatrics 1998;102:11781184.

40. Harvold E, Tomer B, Varggesik K, et al. Primate experiments on oral respiration. Am J Orthod 1981;79:359-372.

41. Traeger N, Schultz B, Pollock AN, et al. Polysomnographic values in children 2-9 years old: additional data and review of the literature. Pediatr Pulmonol 2005; 40:22-30.

42. American Academy of Sleep Medicine. The international classification of sleep disorders diagnostic and coding manual. $2^{\text {nd }}$ ed. Westchester, II: American Academy of Sleep Medicine, 2005.

43. American Thoracic Society: Standards and indications for cardiopulmonary sleep studies in children. Am J Respir Crit Care Med 1996;153:866-878.

44. Guilleminault C, Pelayo R, Leger D, Clerk A, Bocian RCZ. Recognition of sleep-disordered breathing in children. Pediatrics 1996; 98:871-882. 